\title{
Genetic variation in angiotensin-converting enzyme 2 gene is associated with extent of left ventricular hypertrophy in hypertrophic cardiomyopathy
}

\author{
Lize van der Merwe $\cdot$ Ruben Cloete $\cdot$ Miriam Revera \\ Marshall Heradien - Althea Goosen - Valerie A. Corfield • \\ Paul A. Brink · Johanna C. Moolman-Smook
}

Received: 31 March 2008/Accepted: 5 June 2008/Published online: 17 June 2008

(C) The Author(s) 2008

\begin{abstract}
Hypertrophic cardiomyopathy, a common, inherited cardiac muscle disease, is primarily caused by mutations in sarcomeric protein-encoding genes and is characterized by overgrowth of ventricular muscle that is highly variable in extent and location. This variability has been partially attributed to locus and allelic heterogeneity of the disease-causing gene, but other factors, including unknown genetic factors, also modulate the extent of hypertrophy that develops in response to the defective sarcomeric functioning. Components of the renin-angiotensin-aldosterone system are plausible candidate hypertrophy modifiers because of their role in controlling blood pressure and biological effects on cardiomyocyte hypertrophy.
\end{abstract}

L. van der Merwe

Biostatistics Unit, Medical Research Council of South Africa,

Tygerberg, South Africa

R. Cloete · V. A. Corfield · J. C. Moolman-Smook $(\square)$

MRC Centre for Molecular and Cellular Biology, Department of Biomedical Sciences,

University of Stellenbosch Health Sciences Faculty,

PO Box 19063, Room 4036, Teaching Block,

Francie van Zijl Drive, Tygerberg 7505, South Africa

e-mail: hm@sun.ac.za

\section{Revera}

Department of Cardiology, IRCCS San Matteo Hospital, Pavia, Italy

M. Heradien · A. Goosen · P. A. Brink

Department of Medicine, University of Stellenbosch Health

Sciences Faculty, Tygerberg, South Africa

\section{Introduction}

We investigated four Single Nucleotide Polymorphisms in the gene-encoding angiotensin converting enzyme 2 (ACE2), a gene on the X-chromosome whose RAAS product modulates cardiac structure and function, for association with hypertrophic traits in 227 individuals belonging to 22 HCM families with known founder disease-causing mutations.

After adjustment for hypertrophy-influencing factors, including the particular disease-causing mutation, we find association between the G-allele of rs879922 and increased left ventricular mass (effect size: $18.7 \mathrm{~g}$ ), maximum interventricular septal thickness (effect size: $1.9 \mathrm{~mm}$ ) as well as maximum posterior wall thickness (effect size: $0.7 \mathrm{~mm}$ ).

These data demonstrate that ACE2 plays a role in modifying the extent of hypertrophy that develops in HCM, independent of the effect of the disease-causing mutation or blood pressure.

Hypertrophic cardiomyopathy (HCM; OMIM \#192600) is a primary cardiac muscle disorder classically caused by mutations (>400) in genes encoding components of the thick and thin filaments of the cardiac sarcomere (http:// genetics.med.harvard.edu/ seidman/cg3/index.html). Extreme variability of the hypertrophic phenotype is a hallmark feature of HCM and has been attributed in part to allelic effects of the disease-causing gene. Evidence from human and transgenic animal studies indicates that the degree of hypertrophy that develops in response to the causal mutation is modified by environmental factors (including demographic factors such as age, sex and body size), as well as by other genetic factors (Semsarian et al. 2001).

Genes within the renin-angiotensin-aldosterone system (RAAS) have been investigated as modifiers of the hypertrophic variability in HCM in recent years (Marian 
2002; Ortlepp et al. 2002), and much emphasis has been placed on the possible role of the angiotensin-convertingenzyme (ACE) I in the development of hypertrophy. However, subsequently, a second ACE, ACEII, has been discovered and its product, viz. angiotensin-(1-7), has been found to antagonize the vasoconstriction mediated by its substrate, angiotensin II. Lieb et al. found that minor alleles of four single nucleotide polymorphisms (SNPs) in the Xchromosomally located ACEII gene (ACE2) were associated with increases in hypertrophic parameters, viz. left ventricular mass indexed to body size (LVMI), as well as end-diastolic interventricular septal wall thickness (IVS) in males, but not in females (Lieb et al. 2006). Subsequently, Wang et al. also reported that minor alleles of other SNPs in ACE2 were again associated with increased IVS in male but not female HCM patients (Wang et al. 2008). In the latter study, it is possible that the effect of the unknown HCM-causing mutations in the case cohort could confound the results. Hence, we aimed to investigate the role of $A C E 2$ variants in the extent of hypertrophy that develops in genotype-known HCM patients belonging to families in which any of three South African HCM founder mutations segregate.

\section{Methods}

\section{Subjects}

The study was approved by the University of Stellenbosch Health Sciences Faculty's Institutional Review Board (N04/03/062). Twenty-two consecutively referred index cases bearing one of the previously reported South African HCM-founder mutations (7 $\mathrm{R}^{2} \mathrm{~W}_{T N N T 2}, 3 \mathrm{R} 403 \mathrm{~W}_{M Y H 7}$ and $11 \mathrm{~A}_{\left.797 \mathrm{~T}_{M Y H 7}\right)}$ were identified and pedigree tracing was performed (Revera et al. 2008). Individuals who gave informed written consent were tested for carrier-status for each of the three founder mutations, as previously described (Moolman-Smook et al. 1999).

\section{Clinical evaluation}

Blood pressure was taken, twice: in the sitting position, after $5 \mathrm{~min}$ of bed rest, and the second measurement was used. Individuals were coded as hypertensive if they had systolic blood pressure $\geq 140 \mathrm{~mm} \mathrm{Hg}$, or diastolic blood pressure $\geq 90$, or were on anti-hypertensive medication. Medical history, and demographic data (age, sex, height, weight) were recorded for each participant. We performed standard electrocardiography on a MAC1200ST after $5 \mathrm{~min}$ of rest in the supine position, and from this derived resting heart rate. Echocardiography was performed anew on all participating individuals, using a standardized procedure to measure the wall thickness in 16 left ventricular wall segments, by a single experienced echocardiographer (MR) who was blinded to mutation status, according to recommendations of the American Society of Echocardiography (Schiller et al. 1989), as previously described (Revera et al. 2008). LVM was calculated using the American Society of Echocardiography's recommended formula for estimation of LVM from 2D-LV linear dimensions:

$\mathrm{LVM}=0.8$

$$
\begin{aligned}
& \times\left(1.04\left[(\text { LVIDd }+ \text { PWTd }+ \text { SWTd })^{3}-(\text { LVIDd })^{3}\right]\right) \\
& +0.6 \mathrm{~g} .
\end{aligned}
$$

\section{ACE2 genotyping}

As the LD structure of this (and other) regions of the genome has not been reported in the study population, we relied on density selection of SNPs. Four polymorphisms, viz. rs1978124 (intron 1), rs2285666 (intron 3), rs879922 (intron 11) and rs4646179 (exon 16), spread through the length of $A C E 2$ at an average spacing of $\sim 8 \mathrm{~kb}$, were selected from dbSNP. These SNPs were chosen because they had been validated by frequency, had been found associated with cardiovascular traits in previous studies (Lieb et al. 2006; Liu et al. 2005; Yang et al. 2006) and were amenable to genotyping by means of restriction enzyme analysis.

ACE2 genomic regions containing the variants of interest were PCR amplified from subjects' genomic DNA using standard methodology (Moolman-Smook et al. 1999); primers and annealing temperature used are given in Table 1. Variants were genotyped by allele-specific restriction enzyme digestion of the PCR amplicons and fragment sizes detected by gel electrophoresis (Table 1). Two independent observers, blinded to phenotypic data, performed allele-calling. Ten percent of samples, randomly selected for each amplicon, were nucleotide sequenced to verify genotyping results. Furthermore, Mendelian inheritance within families was assessed by Pedstats, using Xchromosome settings, and inconsistencies were resolved by re-genotyping.

Statistical analyses

Quantile normalization was used to convert each trait to approximate normality (Pilia et al. 2006). Associations of $A C E 2$ variants with heritable hypertrophy traits, viz. left ventricular mass (LVM), maximum interventricular septal thickness (maxIVS) and maximum posterior wall thickness (maxPW), were assessed with specialized mixed-effects models utilizing per-individual random effects which are correlated according to kinship coefficients (R package 
Table 1 Summary of PCR-based allele-specific restriction enzyme analysis used for genotyping of ACE2 variants

\begin{tabular}{lllllll}
\hline Variant & Primers $\left(5^{\prime}-3^{\prime}\right)$ & $\begin{array}{l}\text { Restriction } \\
\text { enzyme }\end{array}$ & $\begin{array}{l}\text { Amplicon } \\
\text { size (bp) }\end{array}$ & $\begin{array}{l}\text { Major allele } \\
\text { sizes (bp) }\end{array}$ & $\begin{array}{l}\text { Minor allele } \\
\text { sizes (bp) }\end{array}$ & Detection method \\
\hline rs1978124 & $\begin{array}{l}\text { F: tcttctggctccttctcagc } \\
\text { R: accacaatggcagagaaaggg }\end{array}$ & Sau96I & 1,044 & $\mathrm{~T}(1044)$ & $\mathrm{C}(962,82)$ & $1.5 \%$ Agarose electrophoresis \\
rs2285666 & $\begin{array}{l}\text { F: gttgtaacccagataatcc } \\
\text { R: gttgaaacacacatatctgc }\end{array}$ & AluI & 130 & $\mathrm{C}(130)$ & $\mathrm{T}(78,52)$ & $12 \%$ PAGE \\
rs879922 & $\begin{array}{l}\text { F: ttgtgttaagatcttgtcc } \\
\text { R: aataaactgagctccagc }\end{array}$ & BfaI & 188 & $\mathrm{C}(188)$ & $\mathrm{G}(146,42)$ & $12 \%$ PAGE \\
rs4646179 & $\begin{array}{l}\text { F: tacagggaggaggatgtgcg } \\
\text { R: tgatggcaatacctgtccacg }\end{array}$ & HpyCH4IV & 680 & $\mathrm{~T}(328,229,123)$ & $\mathrm{C}(328,152,123,77)$ & $2.5 \%$ Agarose electrophoresis \\
\hline
\end{tabular}

$b p$ base pairs, $P A G E$ polyacrylamide gel electrophoresis

kinship, function lmekin). These models include variance components for environment and polygenes. Furthermore, concomitant adjustment was made for factors known to influence cardiac hypertrophy, viz. age, sex, body surface area, heart rate, systolic and diastolic blood pressure, hypertension diagnosis, as well as the identity of the disease-causing mutation, by including these as covariates in the analyses. We then formally tested the interaction between sex and genotype, after modeling the genotypes as the number of minor alleles (additive term), and a dominance term, which was only non-zero for heterozygous females (Cordell and Clayton 2005). This model is equivalent to modeling the genotypes as categories, labeling the male genotypes differently to the female genotypes. When the interaction between sex and genotype was not statistically significant, it was discarded from the model. The dominance term was similarly discarded if not significantly different from zero. The resulting models are summarized and discussed.

As effect sizes derived from analysis of transformed data cannot be interpreted in terms of the original measurement units, we also modeled the raw data in terms of the genotypes of the associated variant rs879922, with adjustment for confounders and to obtain informative effect sizes. Because there were only two GG homozygotes in the group, we combined them with the heterozygotes to estimate the effect of at least one G-allele.

Linkage disequilibrium between the four markers and Hardy-Weinberg equilibrium in the study cohort was assessed by Haploview vs3.32.

\section{Results}

Three hundred and twenty individuals belonging to 7 $\mathrm{R}_{22} \mathrm{~W}_{T N N T 2}, 3 \mathrm{R}_{403 \mathrm{~W}_{M Y H 7}}$ and $12 \mathrm{~A}_{797 \mathrm{~T}_{M Y H 7}}$ families were genotyped for variants in $A C E 2$; of these, 227 individuals, including all HCM mutation-carriers, consented to clinical investigation; clinical and demographic traits of these individuals are summarized in Table 2.

All markers were in Hardy-Weinberg equilibrium in the unrelated individuals and there was no relevant correlation between the SNPs (all $r^{2} \leq 0.1$ ). Genotype frequencies of the markers in the study population are given in Table 3 .

Statististically, there were no significant interactions between sex and genotype, so that no sex-specific effects of alleles were observed (results not shown). As the dominance term was not significantly different from zero, we could model the genotypes as single numerical factors, specified as the number of minor-alleles carried by the individual; thus males had a maximum of one of these alleles and females a maximum of two ("additive" coding) (Cordell and Clayton 2005). The $P$ values for significance

Table 2 Basic statistics of relevant clinical and demographic traits in the study cohort, stratified by HCM-mutation status

\begin{tabular}{|c|c|c|c|c|}
\hline & \multirow{2}{*}{\multicolumn{2}{|c|}{$\begin{array}{l}\text { HCM-mutation bearers } \\
N=127\end{array}$}} & \multirow{2}{*}{\multicolumn{2}{|c|}{$\begin{array}{l}\text { Controls } \\
N=100\end{array}$}} \\
\hline & & & & \\
\hline & Median & $(\mathrm{Q} 1, \mathrm{Q} 3)$ & Median & (Q1,Q3) \\
\hline Age (years) & 41 & $(25,53)$ & 41 & $(29,51)$ \\
\hline BSA $\left(\mathrm{kg} / \mathrm{m}^{2}\right)$ & 1.8 & $(1.6,2.0)$ & 1.8 & $(1.7,2.0)$ \\
\hline SystBP (mm Hg) & 120 & $(110,130)$ & 120 & $(110,130)$ \\
\hline DiastBP (mm Hg) & 80 & $(70,80)$ & 80 & $(70,90)$ \\
\hline Heart rate (bpm) & 66 & $(60,75)$ & 69 & $(62,76)$ \\
\hline $\operatorname{LVM}(\mathrm{g})$ & 166 & $(125,218)$ & 126 & $(103,158)$ \\
\hline mIVST (mm) & 13.9 & $(10.4,19.6)$ & 10.2 & $(9.0,11.3)$ \\
\hline $\mathrm{mPW}(\mathrm{mm})$ & 10.1 & $(9.0,11.6)$ & 9.0 & $(8.0,9.7)$ \\
\hline
\end{tabular}

Because of the skewness of some distributions, data are summarised here as median (interquartile range), and were quantile normalized prior to association analysis

$\left(\mathrm{Q}_{1}, \mathrm{Q}_{3}\right)=\mathrm{Q}_{1}$, first quartile, $\mathrm{Q}_{3}$, third quartile; BSA, body surface area; Syst, systolic; BP, blood pressure; Diast, diastolic; bpm, beats per minute; LVM, left ventricular mass; g, grams; mIVST, maximum interventricular septal thickness; mm, millimeter; mPW, maximum posterior wall thickness 
Table 3 Genotype frequencies for ACE2 variants in the complete study cohort, stratified by gender

\begin{tabular}{lllllll}
\hline & \multicolumn{2}{l}{ Male } & & & \multicolumn{2}{l}{ Female } \\
\cline { 2 - 3 } & C & G & & CC & CG & GG \\
\hline rs1978124 & 0.38 & 0.62 & & 0.16 & 0.31 & 0.53 \\
rs2285666 & 0.81 & 0.19 & & 0.59 & 0.38 & 0.03 \\
rs879922 & 0.77 & 0.23 & & 0.50 & 0.49 & 0.01 \\
rs4646179 & 0.98 & 0.02 & & 0.98 & 0.02 & 0.00 \\
\hline
\end{tabular}

tests of the additive term, after removing the dominance term from the model, are shown in Table 4. While there was no evidence for association between hypertrophy traits and ACE2 SNPs rs1978124, rs2285666 or rs4646179, the G-allele of rs879922 was found to have a significant effect on all three of the investigated hypertrophic traits, increasing each by a modest amount. The effect sizes of the G-allele on untransformed, covariate-adjusted traits were $18.7 \mathrm{~g}$ for LVM, $1.9 \mathrm{~mm}$ for maxIVS and $0.7 \mathrm{~mm}$ for maxPW (Table 4).

\section{Discussion}

In this study, we used HCM families, in which the disease is caused by defined founder mutations, to assess the role of variants in ACE2 in hypertrophy development, independent of factors that are known to influence this phenomenon. We find that the G-allele of rs879922 is associated with increased hypertrophy, viz. increased LVM, maxIVS and maxPW.

Lieb et al. previously reported association of the rs879922 G-allele, singly as well as within a four-marker haplotype (with rs4646156, rs4240157 and rs233575), with modestly increased LVM and maxIVS in the general male population in Augsburg, Germany (Lieb et al. 2006). Wang et al. recently reported association of other ACE2 variants, rs2106809 and rs663677, with maxIVS, again only in a group of male HCM patients, with unknown primary disease-causing mutations (Wang et al. 2008).
Although ACE2 maps to a hypertension quantitative trait locus identified in three different rat models of hypertension, no association between ACE2 variants and hypertension has been found to date (Benjafield et al. 2004; Lieb et al. 2006; Wang et al. 2008). In our study, association with hypertrophy was also independent of blood pressure per se or diagnosis of hypertension.

Taken together, our data indicate a role for variation in ACE2 in modifying the development of left ventricular hypertrophy. Furthermore, the results suggest that the genetic modifiers of the cardiac hypertrophy that develops in response to a primary HCM-causing mutation may also be involved in hypertrophy development in the general population. The effect size of the G-allele of rs879922 is modest, as has been previously described for other RAAS gene variants (Mayosi et al. 2003), which may suggest that the hypertrophic phenotype is the cumulative result of a number of genes of moderate effect. As rs879922 occurs within an intron and does not have any obvious functional role, it is possible that the association noted in this study, as well as that of Lieb et al., indicates the effect of a functional variant in linkage disequilibrium with rs879922. The extent of variation in this gene and the LD distribution across the genome in the study population is unknown. However, rs879922 tags at least 2-8 additional HAPMAP SNPs in the CEU, YRI and JPT + CHB populations. In the CEU and YRI HAPMAP populations, variation in ACE2 is predominantly captured by two and three haplotype blocks, respectively; rs879922 is located within a haplotype block involving the $3^{\prime}$ approximately third of the gene, which may indicate that the functional variant lies in this region of the gene.

Due to the highly variable distribution of left ventricular hypertrophy in HCM, it is difficult to distinguish a single echocardiographic measure that accurately quantifies the extent of hypertrophy in all patients. Although LVM is most often used to quantify hypertrophy in HCM, it is known to be an inaccurate measure when echocardiographically derived, due to the asymmetric nature of hypertrophy in HCM. Thus, we also investigated hypertrophic traits that

Table 4 Effect size (beta), standard error (SE) and $P$ value for tests of association of variants in ACE2

\begin{tabular}{|c|c|c|c|c|c|c|c|c|c|}
\hline \multirow[t]{2}{*}{ SNP } & \multicolumn{3}{|c|}{ LVMecho } & \multicolumn{3}{|c|}{$\max$ IVST } & \multicolumn{3}{|l|}{$\max P W$} \\
\hline & Beta & SE & $P$ value & Beta & SE & $P$ value & Beta & SE & $P$ value \\
\hline rs1978124 & -8.0 & 5.9 & 0.1190 & -1.14 & 0.62 & 0.2448 & -0.315 & 0.234 & 0.0772 \\
\hline rs2285666 & 6.7 & 8.5 & 0.6544 & 0.02 & 0.80 & 0.7796 & -0.246 & 0.276 & 0.1825 \\
\hline rs879922 & 18.7 & 9.4 & 0.0205 & 1.87 & 0.87 & 0.0393 & 0.657 & 0.305 & 0.0090 \\
\hline rs4646179 & 26.9 & 27.0 & 0.4813 & 3.71 & 2.56 & 0.2999 & 0.154 & 0.893 & 0.6102 \\
\hline
\end{tabular}

Effect size and standard error are given in original units of measurement for traits, for ease of interpretation, while $P$ values are for tests on quantile normalised traits. Beta is the effect on the trait of adding a G-allele

$L V M$ left ventricular mass, maxIVST maximum interventricular septal thickness, $\max P W$ maximum posterior wall thickness 
are heritable (Mayosi et al. 2002) and can be directly measured, viz. maxIVS and maxPW, rather than calculated from geometric assumptions.

We did not employ Bonferroni correction for multiple testing, as the traits investigated are highly correlated, and such correction is considered over-conservative, risking the rejection of important findings, while Bayesian methods for correction rely on knowledge of prior probability of involvement, which is currently unknown for most genetic variants (Campbell and Rudan 2002). However, even if the over-stringent Bonferroni correction for four independent tests (for the four markers) were performed, the association noted for rs879922 with maxPW would remain significant, and that with LVM remain a trend.

Although the number of subjects assessed in this study is modest, our data adds to previous investigations in confirming the involvement of ACE2 in the development of cardiac hypertrophy, independent of blood pressure or indeed other hypertrophy covariates, in HCM patients. Furthermore, in our study, the use of data derived from HCM families carrying known, founder mutations afforded benefits over case-control studies, such as additional control of genotyping accuracy, control over the variability introduced by distinct primary disease-causing mutations, and avoidance of the confounding effect of occult population stratification. Furthermore, our study combined with that of Lieb et al. and Wang et al. supports a more consistent role for the recently identified component of RAAS, ACEII, than the discordant findings for the original ACEI, in modulating cardiac hypertrophy, and in particular in modifying the extent of hypertrophy that develops in response to HCM-causing mutations.

Acknowledgments GE Healthcare (Germany) is thanked for the pro Deo use of a Vivid7 cardiovascular ultrasound system for the duration of the study. This work was funded by the Wellcome Trust (International senior research fellowship GR073610MA to JCM-S) and the South African National Research Foundation (FA2006040300007 to JCM-S.).

Open Access This article is distributed under the terms of the Creative Commons Attribution Noncommercial License which permits any noncommercial use, distribution, and reproduction in any medium, provided the original author(s) and source are credited.

\section{References}

Benjafield AV, Wang WY, Morris BJ (2004) No association of angiotensin-converting enzyme 2 gene (ACE2) polymorphisms with essential hypertension. Am J Hypertens 17:624-628

Campbell H, Rudan I (2002) Interpretation of genetic association studies in complex disease. Pharmacogenomics J 2:349-360

Cordell HJ, Clayton DG (2005) Genetic association studies. Lancet 366:1121-1131
Lieb W, Graf J, Gotz A, Konig IR, Mayer B, Fischer M, Stritzke J, Hengstenberg C, Holmer SR, Doring A, Lowel H, Schunkert H, Erdmann J (2006) Association of angiotensin-converting enzyme 2 (ACE2) gene polymorphisms with parameters of left ventricular hypertrophy in men. Results of the MONICA Augsburg echocardiographic substudy. J Mol Med 84:88-96

Liu TB, Shang HP, Zhang KX, Chen LH, Zhu XL, Zhang Y, Zhu DL, Huang W (2005) Association of angiotensin I converting enzyme 2 gene polymorphism with essential hypertension in Chinese. Zhonghua Yi Xue Yi Chuan Xue Za Zhi 22:569-571

Marian AJ (2002) Modifier genes for hypertrophic cardiomyopathy. Curr Opin Cardiol 17:242-252

Mayosi BM, Keavney B, Kardos A, Davies CH, Ratcliffe PJ, Farrall M, Watkins H (2002) Electrocardiographic measures of left ventricular hypertrophy show greater heritability than echocardiographic left ventricular mass. Eur Heart J 23:1963-1971

Mayosi BM, Keavney B, Watkins H, Farrall M (2003) Measured haplotype analysis of the aldosterone synthase gene and heart size. Eur J Hum Genet 11:395-401

Moolman-Smook JC, De Lange WJ, Bruwer EC, Brink PA, Corfield VA (1999) The origins of hypertrophic cardiomyopathy-causing mutations in two South African subpopulations: a unique profile of both independent and founder events. Am J Hum Genet 65:1308-1320

Ortlepp JR, Vosberg HP, Reith S, Ohme F, Mahon NG, Schroder D, Klues HG, Hanrath P, McKenna WJ (2002) Genetic polymorphisms in the renin-angiotensin-aldosterone system associated with expression of left ventricular hypertrophy in hypertrophic cardiomyopathy: a study of five polymorphic genes in a family with a disease causing mutation in the myosin binding protein $\mathrm{C}$ gene. Heart 87:270-275

Pilia G, Chen WM, Scuteri A, Orru M, Albai G, Dei M, Lai S, Usala G, Lai M, Loi P, Mameli C, Vacca L, Deiana M, Olla N, Masala M, Cao A, Najjar SS, Terracciano A, Nedorezov T, Sharov A, Zonderman AB, Abecasis GR, Costa P, Lakatta E, Schlessinger D (2006) Heritability of cardiovascular and personality traits in 6, 148 Sardinians. PLoS Genet 2:e132

Revera M, Van Der ML, Heradien M, Goosen A, Corfield VA, Brink PA, Moolman-Smook JC (2008) Troponin T and beta-myosin mutations have distinct cardiac functional effects in hypertrophic cardiomyopathy patients without hypertrophy. Cardiovasc Res 77:687-694

Schiller NB, Shah PM, Crawford M, DeMaria A, Devereux R, Feigenbaum H, Gutgesell H, Reichek N, Sahn D, Schnittger I (1989) Recommendations for quantitation of the left ventricle by two-dimensional echocardiography. American Society of Echocardiography Committee on Standards, Subcommittee on Quantitation of Two-Dimensional Echocardiograms. J Am Soc Echocardiogr 2:358-367

Semsarian C, Healey MJ, Fatkin D, Giewat M, Duffy C, Seidman CE, Seidman JG (2001) A polymorphic modifier gene alters the hypertrophic response in a murine model of familial hypertrophic cardiomyopathy. J Mol Cell Cardiol 33:2055-2060

Wang SX, Fu CY, Zou YB, Wang H, Shi Y, Xu XQ, Chen JZ, Song XD, Huan TJ, Hui RT (2008) Polymorphisms of angiotensinconverting enzyme 2 gene associated with magnitude of left ventricular hypertrophy in male patients with hypertrophic cardiomyopathy. Chin Med J (Engl) 121:27-31

Yang W, Huang W, Su S, Li B, Zhao W, Chen S, Gu D (2006) Association study of ACE2 (angiotensin I-converting enzyme 2) gene polymorphisms with coronary heart disease and myocardial infarction in a Chinese Han population. Clin Sci (Lond) 111:333-340 